\title{
Identification of Vortex Cores in Cerebral Aneurysms on 4D Flow MRI
}

W e read with interest the study by Futami et al, "Identification of Vortex Cores in Cerebral Aneurysms on 4D Flow MRI." They described the visualization, complexity, and stability of vortex cores on $4 \mathrm{D}$ flow MR imaging in a series of 40 cerebral aneurysms. Discriminating single, stable, vortex cores from complex flow patterns with temporarily unstable vortices, they found an association between a complex vortical flow pattern and complex aneurysm morphology. Thus, they concluded that the identification of vortex core flow patterns on $4 \mathrm{D}$ flow MR imaging may help to stratify aneurysm rupture risk. Moreover, they explained the mathematic definition of a vortex core line and referred to other previous research using computational fluid dynamics simulation data models that describe the phenomenon of vortex cores with cerebral aneurysm models. ${ }^{2,3}$

We agree with Futami et $\mathrm{al}^{1}$ that vortex core identification is a promising new tool for aneurysm assessment, which is in line with our findings published more than 10 years ago. ${ }^{4}$ Our group first described normal intracranial artery hemodynamics with a helical flow phenomenon in the carotid siphon using in vivo $4 \mathrm{D}$ flow $\mathrm{MR}$ imaging. ${ }^{5}$ Moreover, we were the first to describe intra-aneurysmal hemodynamics, including the visualization of intra-aneurysmal vortex cores. ${ }^{4}$ In these studies, we performed postprocessing and visualization of aneurysm flow using a commercially available $3 \mathrm{D}$ visualization software package (EnSight; Computational Engineering International, Apex, North Carolina). Besides 3D streamlines, 2D vector graphs, and time-resolved particle traces, a dedicated integrated algorithm of this software was applied to segment and illustrate vortex core patterns from $3 \mathrm{D}$ vector fields. Thus, the core of the vortical is represented by colored bars indicating the location and magnitude of the vortical flow.

We were also able to analyze the temporal and spatial stability of vortex cores inside the aneurysm during the whole cardiac cycle (Fig 3 from our original work ${ }^{4}$ ). Likewise in the work by Futami et $\mathrm{al}^{1}{ }^{1} \mathrm{a}$ link between the stability and complexity of intra-aneurysmal vortex core patterns and aneurysm geometry, including the aspect ratio, was disclosed in a series of 5 cerebral aneurysms. In 2 of these aneurysms with a smooth surface and low aspect ratio, temporally stable, single vortex cores were identified, whereas in the other aneurysms with a lobulated surface and/or high aspect ratio, vortex cores showed spatiotemporal and magnitude alterations during the cardiac cycle.
We acknowledge the authors' work studying vortex core patterns in a higher number of aneurysms and support their conclusions drawn from their cohort, though mostly ruptured cases were included. However, we would like to stress that correct reference to the existing body of literature should be given relative to the original description of in vivo visualization of aneurysmal vortex core flow.

Disclosures: Michael Markl—UNRELATED: Consultancy: Circle Cardiovascular Imaging; Grants/Grants Pending: research support: Siemens; research grant, Circle Cardiovascular Imaging; research grant, Cryolife.* *Money paid to the institution.

\section{REFERENCES}

1. Futami K, Uno T, Misaki K, et al. Identification of vortex cores in cerebral aneurysms on $4 \mathrm{D}$ flow MRI. AJNR Am J Neuroradiol 2019;40:2111-16 CrossRef Medline

2. Le TB, Troolin DR, Amatya D, et al. Vortex phenomena in sidewall aneurysm hemodynamics: experiment and numerical simulation. Ann Biomed Eng 2013;41:2157-70 CrossRef Medline

3. Oeltze-Jafra S, Cebral JR, Janiga G, et al. Cluster analysis of vortical flow in simulations of cerebral aneurysm hemodynamics. IEEE Trans Vis Comput Graph 2016;22:757-66 CrossRef Medline

4. Meckel S, Stalder AF, Santini F, et al. In vivo visualization and analysis of 3-D hemodynamics in cerebral aneurysms with flow sensitized 4-D MR imaging at 3 T. Neuroradiology 2008;50:473-84 CrossRef Medline

5. Wetzel S, Meckel S, Frydrychowicz A, et al. In vivo assessment and visualization of intracranial arterial hemodynamics with flow-sensitized 4D MR imaging at 3T. AJNR Am J Neuroradiol 2007;28:433-38 Medline Medline

(D) Meckel Department of Neuroradiology Faculty of Medicine Medical Center, University of Freiburg Freiburg, Germany

(1) M. Markl Departments of Radiology and Biomedical Engineering Feinberg School of Medicine and McCormick School of Engineering Northwestern University Chicago, Illinois

(D) Wetzel Institute of Neuroradiology Hirslanden Clinic Zurich Zurich, Switzerland 